\title{
Sliding Mode Controller for Trajectory Tracking Control of Autonomous Mobile Robot
}

\author{
Ang Oon Thay, Mohd Ariffanan Mohd Basri*, Nurul Adilla Mohd Subha, Mohamad Amir Shamsudin \& \\ Shafishuhaza Sahlan
}

Faculty of Electrical Engineering, Universiti Teknologi Malaysia, 81310 UTM Skudai, Johor, Malaysia.

*Corresponding author: ariffanan@fke.utm.my

\begin{abstract}
Trajectory tracking control is an important issue in the field of autonomous mobile robot. In high speed and heavy load applications, the dynamic of autonomous mobile robot plays an important factor in allowing the robot to follow the desired trajectory path. However, the parameters attribute to robot dynamic are difficult to model and highly uncertain. One of the uncertainty factors is the load variation which changes the dynamic parameters of autonomous mobile robot. Meanwhile, Sliding Mode Control (SMC) is well known for its robustness against model uncertainties and disturbances. In this paper, design of dynamic controller based on SMC technique for trajectory tracking control of autonomous mobile robot system is presented. The model of mobile robot is developed based on Pioneer 3-DX mobile robot. The trajectory tracking controller is divided into two parts, kinematic controller and dynamic controller. Stability of both dynamic and kinematic controller is verified using Lyapunov stability theory. The performance of trajectory tracking control for proposed dynamic controller based on SMC technique is compared against dynamic controller based on Proportional-Integral-Derivative (PID) technique with and without the presence of dynamic uncertainties. Simulation results show that the proposed dynamic controller based on SMC technique yields better performance in trajectory tracking control in comparison to PID technique.
\end{abstract}

Keywords: Autonomous mobile robot, robust control, sliding mode control, trajectory tracking.

Article History: received 30 May 2017; accepted 11 April 2018; published 16 April 2018.

(0) 2018 Penerbit UTM Press. All rights reserved.

\section{INTRODUCTION}

Currently, autonomous mobile robot is widely used in industries such as defense and rescue operations, transportation in warehouses, space exploration, inspection in constrained spaces, and services such as office, restaurants, fire and security, people with disabilities, etc. Among different mobile robot structure, unicycle-like mobile robots are frequently adopted due to its simple configuration and good mobility.

For several decades, trajectory tracking control has been one of the active research areas in mobile robots, specifically for under-actuated mobile robots such as unicycle-like mobile robot [1]. Trajectory tracking control enables mobile robot to follow its time parameterized reference path and reach its destination i.e., a geometric path with an associated timing law. This is very important for mobile robot in accomplishing a task [2]. Studies in early stage for trajectory tracking control considers only robot kinematic [3, 4]. In such cases, the vehicle control inputs are calculated by assuming that there is perfect velocity tracking which the robot follows requiring trajectory without velocity error [5].

Unfortunately, robot dynamic exists in real application. Practically, considering solely on robot kinematic is insufficient to produce a good trajectory tracking performance specifically in high speed and heavy load application [6]. Hence, throughout the years researchers have integrated both kinematic controller and dynamic controller as their trajectory tracking controller. However, robot dynamic is rather complicated since it involves a number of parameters such as mass, centre of gravity, force, torque, speed, acceleration and moment of inertia. These parameters are highly uncertain and vary with times. Thus, it is practically impossible to model robot dynamic accurately. To compensate this uncertainty factor in robot dynamic, researchers have introduced several control techniques which include fuzzy control [7, 8], neural network [9], adaptive control [2, 10], etc. Significantly, among others, sliding mode control (SMC) provides a robust control technique for nonlinear system which offers great robustness against uncertainties, small tracking error and fast response [11].

In this paper, design of dynamic controller using SMC technique for trajectory tracking control of autonomous mobile robot is presented. The work is carried out using MATLAB software. The model of mobile robot is based on Pioneer 3-DX mobile robot. It is developed based on work executed in [12]. The trajectory tracking controller is divided into two parts, kinematic controller and dynamic controller. The kinematic controller is adopted from [2] to generate a desired velocity command based on current robot position and desired position. As for dynamic controller, two different controllers are developed. First, is the proposed dynamic controller based on SMC technique and the other is a conventional controller based on 
Proportional-Integral-Differentiate (PID) technique [13]. These dynamic controllers are used to compensate the robot dynamic effects. Finally, the mobile robot is simulated to follow both eight-shaped and circle-shaped reference trajectories at a desired speed of $0.25 \mathrm{rad} / \mathrm{s}$. The trajectory tracking performance for both robots dynamic controllers are evaluated with and without the presence of dynamic uncertainties in the system.

\section{UNICYCLE_LIKE MOBILE ROBOT MODEL}

A unicycle-like mobile robot structure is illustrated in Figure 1, where $\mathrm{G}$ is the center of mass of the robot; $\mathrm{B}$ is center of wheel baseline; $\mathrm{C}$ is the position of the castor wheel; $\mathrm{E}$ is the location of a tool onboard the robot; $\mathrm{h}=[\mathrm{x}$ $y]^{\mathrm{T}}$ is the point of interest to tracks a trajectory; $\mathrm{a}, \mathrm{b}, \mathrm{c} \mathrm{d}, \mathrm{e}$ are the distances between wheel baseline and its corresponding points as illustrated in Figure 1. Meanwhile, $\mu$ is the linear velocity of mobile robot, $\omega$ is the angular velocity of mobile robot, and $\psi$ is the orientation of mobile robot with respects to point $h$.

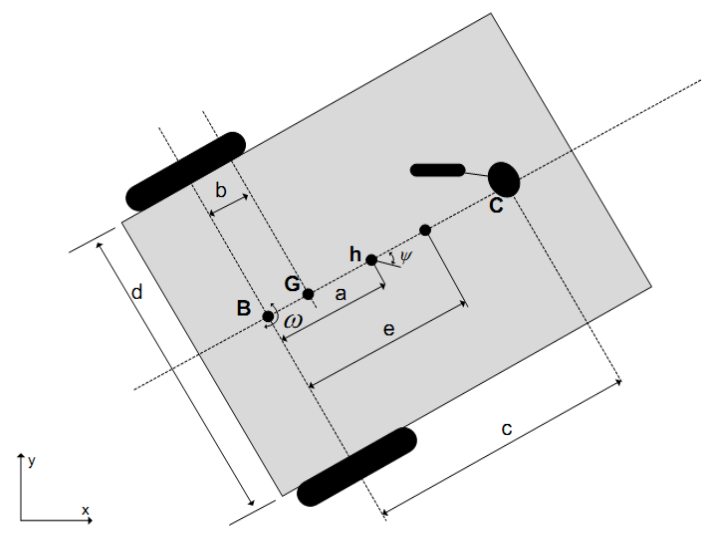

Figure 1. Structure of unicycle-like mobile robot

By applying the fundamental physic and mathematic laws, the complete model of unicycle-like mobile robot can be written as shown in (1). This model is derived by De La Cruz and Carelli [12] where it included both kinematic and dynamic models of unicycle-like mobile robot. The symbol $\mathrm{x}$ represents the derivative of coordinate $\mathrm{x}$ in $\mathrm{XY}$ plane; $\mathrm{y}$ is the derivative of coordinate $\mathrm{y}$ in $\mathrm{XY}$ plane; is the derivative of mobile robot orientation, $\psi ; \iota \&$ and $u$ are the derivative of linear velocity, $u$ and angular velocity, $w$ respectively; $u_{\text {ref }}$ and $w_{\text {ref }}$ are the desired values of the linear and angular velocities, respectively. They are also represent the input signals of the system; [ $\theta_{1}$ $\left.\theta_{2} \theta_{3} \theta_{4} \theta_{5} \theta_{6}\right]^{\mathrm{T}}$ is the vector of dynamic model parameters of mobile robot; [ $\left.\delta_{x} \delta_{y} 0 \delta_{\mu} \delta_{\omega}\right]^{\mathrm{T}}$ represent the vector of uncertainties that may presents in the system.

$$
\left[\begin{array}{c}
\not \& \\
\& \& \\
\psi \& \\
\iota \& \\
u \&
\end{array}\right]=\left[\begin{array}{c}
u \cos \psi-a w \sin \psi \\
u \sin \psi+a w \cos \psi \\
w \\
\frac{\theta_{3}}{\theta_{1}} w^{2}-\frac{\theta_{4}}{\theta_{1}} u \\
-\frac{\theta_{5}}{\theta_{2}} u w-\frac{\theta_{6}}{\theta_{2}} w
\end{array}\right]+\left[\begin{array}{cc}
0 & 0 \\
0 & 0 \\
0 & 0 \\
\frac{1}{\theta_{1}} & 0 \\
0 & \frac{1}{\theta_{2}}
\end{array}\right]\left[\begin{array}{l}
u_{r e f} \\
w_{r e f}
\end{array}\right]+\left[\begin{array}{l} 
\\
\delta_{x} \\
\delta_{y} \\
0 \\
\delta_{\mu} \\
\delta_{\omega}
\end{array}\right]
$$

According to [12], the dynamic parameters $\theta_{i}$ can be represented by (2) - (7). The elements present in the dynamic parameter $\theta_{i}$ are the physical parameters of the mobile robot, such as robot mass m, moment of inertia $I_{z}$ at $\mathrm{G}$ about $\mathrm{y}$ axis, $R_{a}$ is electrical resistance of robot motors, $k_{b}$ is the electromotive constant of robot motors, $k_{a}$ is the constant of torque of its motors, $r$ the radius of the wheels, $\mathrm{b}$ and $\mathrm{d}$ are the distances (refer Figure 1), $B_{e}$ and $I_{e}$ are friction and moment of inertia of combined motor rotor, gearbox and wheel, and $R_{t}$ is the nominal radius of tire.

$$
\begin{aligned}
\theta_{1} & =\left[\frac{R_{a}}{k_{a}}\left(m R_{t} r+2 I_{e}\right)+2 r k_{D T}\right] \frac{1}{2 r k_{P T}} \\
\theta_{2} & =\left[\frac{R_{a}}{k_{a}}\left(I_{e} d^{2}+2 r^{2}\left(I_{z}+m b^{2}\right)\right)+2 r d k_{D R}\right] \frac{1}{2 r d k_{P R}} \\
\theta_{3} & =\frac{R_{a}}{k_{a}} \frac{m b R_{t}}{2 k_{P T}} \\
\theta_{4} & =\frac{R_{a}}{k_{a}}\left(\frac{k_{a} k_{b}}{R_{a}}+B_{e}\right) \frac{1}{r k_{P T}}+1 \\
\theta_{5} & =\frac{R_{a}}{k_{a}} \frac{m b R_{t}}{d k_{P R}} \\
\theta_{6} & =\frac{R_{a}}{k_{a}}\left(\frac{k_{a} k_{b}}{R_{a}}+B_{e}\right) \frac{1}{2 r k_{P R}}+1
\end{aligned}
$$

The robot model in (1) has incorporated PD velocity controllers to control its motors. This is to ease the integration of designed controller for commercial robots. The proportional gains of the controller are $k_{P T}$ and $k_{P R}$, and derivative gains of the controllers are $k_{D R}$ and $k_{D T}$.

\section{TRAJECTORY TRACKING CONTROLLER DESIGN}

The design of trajectory tracking controller is divided into two parts. First is the kinematic controller to generate the desired linear and angular velocities based on differences between desired position $\left(\mathrm{x}_{\mathrm{d}}, \mathrm{y}_{\mathrm{d}}\right)$ and robot current position $(\mathrm{x}, \mathrm{y})$. The kinematic controller design is adopted from [2]. The second part is the dynamic controller design. It is used to compensate the dynamic effects of mobile robot. The complete structure of trajectory tracking controller is shown in Figure 2. Notice this structure has been presented in [2] and [12]. 


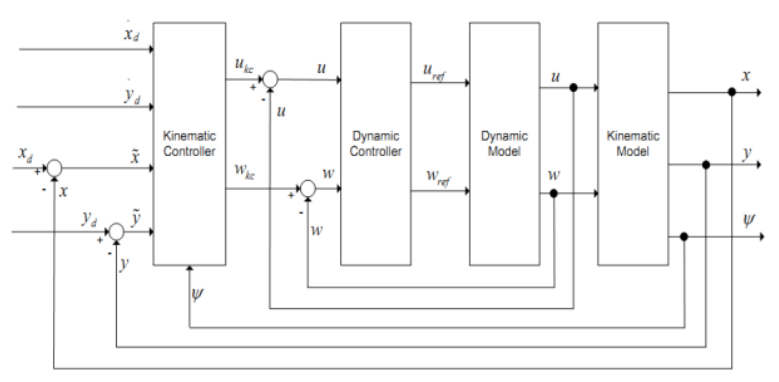

Figure 2. Structure of proposed trajectory tracking controller

\subsection{Kinematic Controller Design and Language}

The objective of kinematic controller is to generate reference linear and angular velocities to dynamic controller as shown in Figure 2. It is designed based on the kinematic model of mobile robot given in (1). Assuming the uncertainties term $\left[\delta_{x} \delta_{y} 0 \delta_{\mu} \delta_{\omega}\right]^{\mathrm{T}}$ in (1) are zero vector, the kinematic model of robot is given by

$$
\left[\begin{array}{l}
\& \\
\& \\
\psi
\end{array}\right]=\left[\begin{array}{cc}
\cos \psi & -a \sin \psi \\
\sin \psi & a \cos \psi \\
0 & 1
\end{array}\right]\left[\begin{array}{l}
u \\
w
\end{array}\right]
$$

From the kinematic model in (8), the inverse kinematic model of robot can be expressed as in (9).

$$
\left[\begin{array}{l}
u \\
w
\end{array}\right]=\left[\begin{array}{cc}
\cos \psi & \sin \psi \\
\frac{-1}{a} \sin \psi & \frac{1}{a} \cos \psi
\end{array}\right]\left[\begin{array}{l}
\& \\
\&
\end{array}\right]
$$

Applying kinematic control law from work in [2], the controller is given by

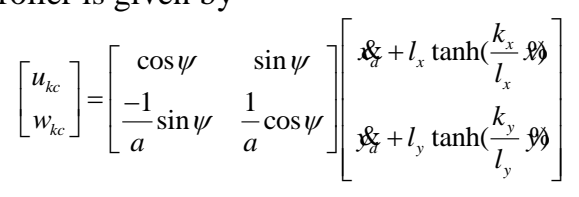

where $l_{x} \in \mathbf{R}$ and $l_{y} \in \mathbf{R}$ are saturation constant; $k_{x}$ and $k_{y}$ are the kinematic controller gain; $\ell \theta=x_{d}-x$ and $f /=y_{d}-y$ are the position error in axis $\mathrm{X}$ and $\mathrm{Y}$ respectively.

\subsection{Stability Analysis of Kinematic Controller}

In analysis the stability of kinematic controller for trajectory tracking control, the effect of robot dynamics are ignored. The velocity tracking is perfect where $u=u_{k c}$ and $w=w_{k c}$. Based on this assumption, equating (9) and (10) would yield the close loop equation in term of velocity error as shown in (11).

$$
\left[\begin{array}{l}
\dot{b} \\
y
\end{array}\right]+\left[\begin{array}{ll}
l_{x} & 0 \\
0 & l_{y}
\end{array}\right]\left[\begin{array}{c}
\tanh \left(\frac{k_{x}}{l_{x}}\right. \\
\tanh \left(\frac{k_{y}}{l_{y}} f y\right.
\end{array}\right]=\left[\begin{array}{l}
0 \\
0
\end{array}\right]
$$

which can be written as (12) after rearranging the matrices.

$$
l \underline{l}=\left[l _ { x } \operatorname { t a n h } \left(\frac{k_{x}}{l_{x}} \ell l_{y} \tanh \left(\frac{k_{y}}{l_{y}} \varphi g\right]^{T}\right.\right.
$$

where $h=\left[\begin{array}{l}\ell 0 \\ y d\end{array}\right]$ is the vector of position errors and $h$ is its

derivative. Notice, (12) has a unique equilibrium point at the origin. Therefore, using Lyapunov candidate function

$$
V=\frac{1}{2} h / P 0
$$

and first time derivative it would yield

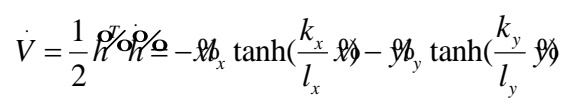

which is negative definite if $l_{x}, l_{y}, k_{x}$ and $k_{y}$ remain positive. This conclude the controlled system using proposed kinematic controller is asymptotically stable at the origin where $f(t) \rightarrow 0$ and $f(t) \rightarrow 0$ as $t \rightarrow \infty$.

\subsection{Dynamic Controller Design based on SMC}

Referring back to the mobile robot model as defined in (1), the dynamic part of mobile robot model can be defined as in (15) after equating the uncertainties term $\left[\delta_{x} \delta_{y} 0 \delta_{\mu}\right.$ $\left.\delta_{\omega}\right]^{\mathrm{T}}$ to zero vector.

$$
\left[\begin{array}{c}
l \& \\
\iota \&
\end{array}\right]=\left[\begin{array}{c}
\frac{\theta_{3}}{\theta_{1}} w^{2}-\frac{\theta_{4}}{\theta_{1}} u \\
-\frac{\theta_{5}}{\theta_{2}} u w-\frac{\theta_{6}}{\theta_{2}} w
\end{array}\right]+\left[\begin{array}{cc}
\frac{1}{\theta_{1}} & 0 \\
0 & \frac{1}{\theta_{2}}
\end{array}\right]\left[\begin{array}{l}
u_{r e f} \\
w_{r e f}
\end{array}\right]
$$

By linear parameterized the dynamic equation (15), it can be written as

$$
\left[\begin{array}{l}
u_{r e f} \\
w_{r e f}
\end{array}\right]=\left[\begin{array}{cccccc}
\iota \& & 0 & -w^{2} & u & 0 & 0 \\
0 & \iota \& & 0 & 0 & u w & w
\end{array}\right]\left[\begin{array}{c}
\theta_{1} \\
\theta_{2} \\
\theta_{3} \\
\theta_{4} \\
\theta_{5} \\
\theta_{6}
\end{array}\right]
$$

which can also be written as

$$
\left[\begin{array}{l}
u_{r e f} \\
w_{r e f}
\end{array}\right]=\left[\begin{array}{cc}
\theta_{1} & 0 \\
0 & \theta_{2}
\end{array}\right]\left[\begin{array}{l}
i \& \\
1
\end{array}\right]+\left[\begin{array}{cccccc}
0 & 0 & -w^{2} & u & 0 & 0 \\
0 & 0 & 0 & 0 & u w & w
\end{array}\right]\left[\begin{array}{c}
\theta_{1} \\
\theta_{2} \\
\theta_{3} \\
\theta_{4} \\
\theta_{5} \\
\theta_{6}
\end{array}\right]
$$

or in compact form,

$$
v_{r e f}=D v+\eta
$$

where $v_{\text {ref }}=\left[\begin{array}{ll}u_{\text {ref }} & w_{\text {ref }}\end{array}\right]^{T}, v=\left[\begin{array}{ll}u & w\end{array}\right]^{T}, D=\operatorname{diag}\left(\theta_{1}, \theta_{2}\right)$ and 


$$
\eta=\left[\begin{array}{cccccc}
0 & 0 & -w^{2} & u & 0 & 0 \\
0 & 0 & 0 & 0 & u w & w
\end{array}\right]\left[\begin{array}{l}
\theta_{1} \\
\theta_{2} \\
\theta_{3} \\
\theta_{4} \\
\theta_{5} \\
\theta_{6}
\end{array}\right]
$$

The design of dynamic controller based on SMC for trajectory tracking control is initiated with definition of sliding surface. Choosing PI type of sliding surface, the sliding surface can be defined as

$$
s(t)=\left[\begin{array}{l}
s_{1}(t) \\
s_{2}(t)
\end{array}\right]=\mathbb{Q}(t)+\beta_{s m c} \int \mathbb{Q}(t)
$$

where $\not(t)$ is vector of linear and angular velocities error, and $\beta_{s m c}$ is vector of sliding surface integral gains. If the system is on the sliding surface where $s(t)=0,(19)$ can be written as $Q k t)=-\beta_{s m c} \int(k t)$. The velocities error will converge to zero, $1 /(00) \rightarrow 0$ if $\beta_{s m c}>0$.

The derivatives of sliding surface function becomes

$$
s(t)=(t)+\beta_{s m c} M(t)
$$

If the system is to maintain on the sliding surface, the derivatives of sliding surface must be equal to zero $\&(t)=0$. Thus, (20) can be written as

$$
\mathscr{L}(t)=\mathbb{L}_{r e f}(t)+\beta_{s m c} 9 \%
$$

Thus, substituting (21) into (18), the equivalent control law can be defined as

$$
v_{r e f}=D\left(\mathbb{R}_{r e f}^{\&}+\beta_{s m c} \mathscr{W}+\eta\right.
$$

Adding term $K_{s m c} \operatorname{sign}(s)$ into (22) for discontinuous control law to account of the uncertainties and disturbance in real application, the control law becomes

$$
v_{r e f}=D\left(\mathbb{R}_{r e f}^{\&}+\beta_{s m c} 900+\eta+K_{s m c} \operatorname{sign}(s)\right.
$$

Taking account of the chattering effect, then control law can be expressed as

$$
v_{r e f}=D\left(\mathbb{R}_{r e f}+\beta_{s m c} \varphi \phi s+\eta+K_{s m c} \frac{s}{|s|+\varepsilon}\right.
$$

where $K_{s m c}$ is vector of switching gains and $\varepsilon$ is the small positive constant.

\subsection{Stability Analysis of Dynamic Controller}

The inverse dynamic model is defined in (18) while the proposed dynamic controller based on SMC is defined in (24). Comparing these two equations, the first time derivative of velocity error is found as shown in equation (25).

$$
D \& \&=D\left(\mathbb{R}_{\text {ref }}+\beta_{s m c} 9 \phi+\eta+K_{s m c} \frac{s}{|s|+\varepsilon}\right.
$$

$$
\begin{aligned}
& \mathscr{L}=\mathbb{L}_{r e f}+\beta_{s m c} \mathscr{1} / \& D^{-1} K_{s m c} \frac{s}{|s|+\varepsilon} \\
& =-\beta_{s m c} \% D^{-1} K_{s m c} \frac{s}{|s|+\varepsilon}
\end{aligned}
$$

Thus, selecting Lyapunov candidate function $V=\frac{1}{2} \% \%$ would obtains its derivative as $1 \&=\%$ \% Therefore, it becomes

$$
V^{\&}=-1 \% \beta_{s m c} \mathscr{H} \% D^{-1} K_{s m c} \frac{s}{|s|+\varepsilon}
$$

From (26), it can be concluded $\&$ negative definite where $1 \&<0$ for $\beta_{s m c}>0, K_{s m c}>0$. Thus, the controlled dynamic system using this dynamic controller based on SMC is asymptotically stable at the origin where $i(t) \rightarrow 0$ and $\mathscr{W}(t) \rightarrow 0$ as $t \rightarrow \infty$.

\section{RESULTS}

In this section, the performance of trajectory tracking control using dynamic controller based on SMC is evaluated for different strength of dynamic uncertainty applied into the system. The different strength of dynamic uncertainty is applied by varying parameters $\theta_{i}$ in robot dynamic model. Table 1 shows the values of parameters $\theta_{i}$ for different strength of uncertainty. For example, $\theta_{1}=0.26$ when there is no uncertainty and increased to 0.29 when $10 \%$ of uncertainty is applied.

Table 1. Values of parameters $\theta_{i}$ at different strength of dynamic uncertainty

\begin{tabular}{|c|c|c|c|c|c|c|}
\hline Case & $\boldsymbol{\theta}_{\boldsymbol{1}}$ & $\boldsymbol{\theta}_{\boldsymbol{2}}$ & $\boldsymbol{\theta}_{\mathbf{3}}$ & $\boldsymbol{\theta}_{\boldsymbol{4}}$ & $\boldsymbol{\theta}_{\mathbf{5}}$ & $\boldsymbol{\theta}_{\boldsymbol{6}}$ \\
\hline $\begin{array}{c}\text { No Un- } \\
\text { certainty }\end{array}$ & 0.26 & 0.25 & $-5.0 \mathrm{e}-4$ & 1.00 & $2.6 \mathrm{e} 3$ & 1.08 \\
\hline $10 \%$ & 0.29 & 0.28 & $-5.5 \mathrm{e}-4$ & 1.10 & $3.0 \mathrm{e} 3$ & 1.18 \\
\hline $30 \%$ & 0.34 & 0.33 & $-6.5 \mathrm{e}-4$ & 1.30 & $3.4 \mathrm{e} 3$ & 1.40 \\
\hline $50 \%$ & 0.39 & 0.38 & $-7.5 \mathrm{e}-4$ & 1.49 & $3.9 \mathrm{e} 3$ & 1.62 \\
\hline
\end{tabular}

The gains of SMC dynamic controller used are $\beta_{s m c}=\left[\begin{array}{cc}\beta_{u} & 0 \\ 0 & \beta_{w}\end{array}\right]=\left[\begin{array}{ll}6 & 0 \\ 0 & 6\end{array}\right] \quad$ and $K_{s m c}=\left[\begin{array}{cc}K_{u} & 0 \\ 0 & K_{w}\end{array}\right]=\left[\begin{array}{cc}0.3 & 0 \\ 0 & 0.3\end{array}\right] \quad$ respectively with $\varepsilon=0.01$.

Figure 3 shows the differences between eight-shaped reference trajectory and real trajectory of mobile robot for different strength of dynamic uncertainty. The mobile robot is able to follow eight-shaped reference trajectory when no dynamic uncertainty is applied. However, the mobile robot is losing its track on reference trajectory as the strength of dynamic uncertainty increases. Figure 4 shows the distance errors of mobile robot in trajectory tracking control at different strength of dynamic uncertainty for eight-shaped trajectory. The large initial distance error is due to the different starting point of 
mobile robot compared to reference trajectory. Based on Figure 4, the distance error reduces rapidly and saturated at a very small value. Hence, indicating that the mobile robot is able to follow the reference trajectory closely as time increases.

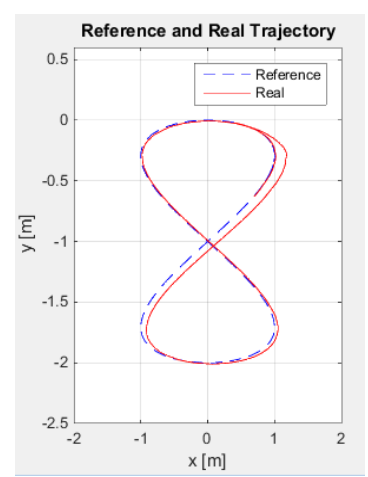

(a)

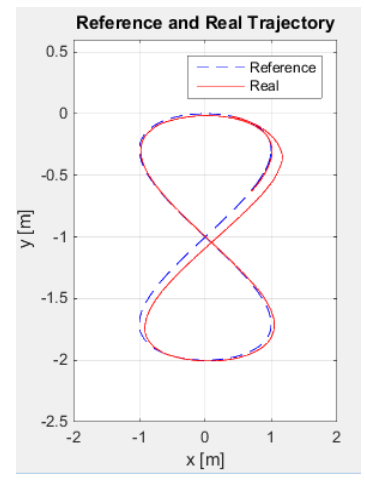

(c)

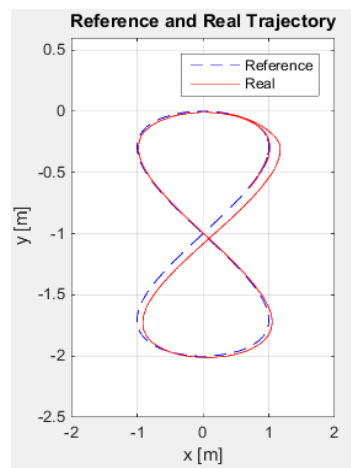

(b)

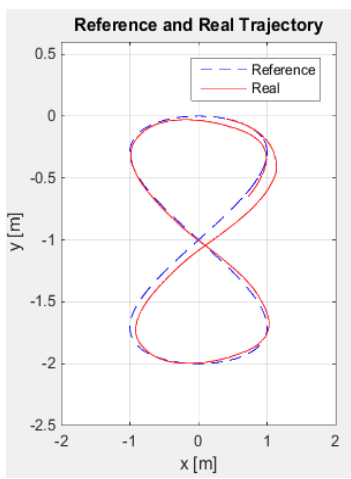

(d)
Figure 3. Reference eight-shaped trajectory and real trajectory of mobile robot using dynamic controller based on SMC at different strength of dynamic uncertainty. a) No uncertainty b) $10 \%$ uncertainty c) $30 \%$ uncertainty d) $50 \%$ uncertainty

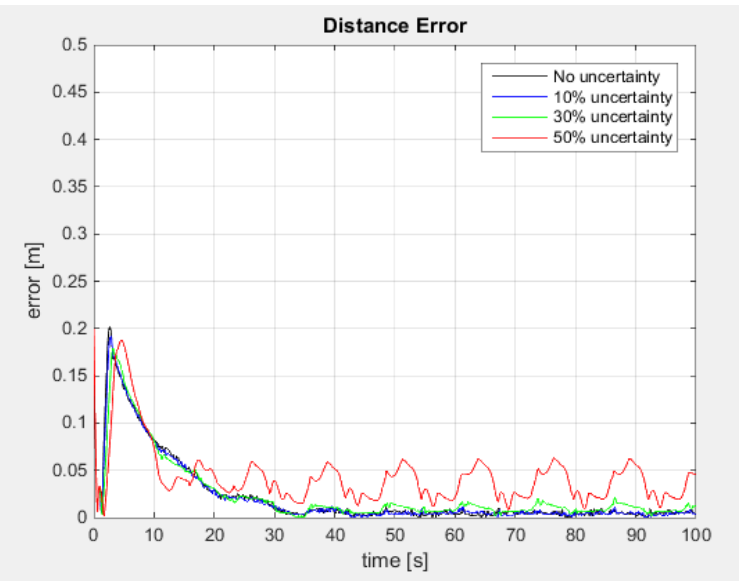

Figure 4. Distance error of trajectory tracking control using dynamic controller based on SMC at different strength of dynamic uncertainty for eight-shaped trajectory

For the purpose of comparison, similar to experiments conducted for dynamic controller based on SMC, the performance of trajectory tracking control using dynamic controller based on PID is evaluated. Figure 5 shows the differences between eight-shaped reference trajectory and real trajectory of mobile robot at different strength of dynamic uncertainty. Notices the mobile robot is able to track the reference trajectory but the distance between them increases trajectory as strength of dynamic uncertainty applied increases. Meanwhile, the distance errors for different strength of dynamic uncertainty are shown as in Figure 6. The distance error means the instantaneous distance differences between desired position and robot position. Based on the results, the distance error is fluctuating throughout the simulation run. This could be due to sharp change of direction of mobile robot when following the eight-shaped reference trajectory. Also, there is exists of high initial error which is due to different starting point between mobile robot and reference trajectory. Nevertheless, the distance error increases proportionally with the increases of dynamic uncertainty applied into the system.

The Integral Absolute Error (IAE) obtained from the simulation tests are tabulated in Table 2. Based on the results, robot tracking on eight-shaped trajectory using SMC has much smaller IAE compared to PID controller. For example, when there is no uncertainty applied, the IAE obtained for eight-shaped trajectory tracking using SMC is $2.1922 \mathrm{~m}$ while the IAE obtained using PID is $5.0530 \mathrm{~m}$.

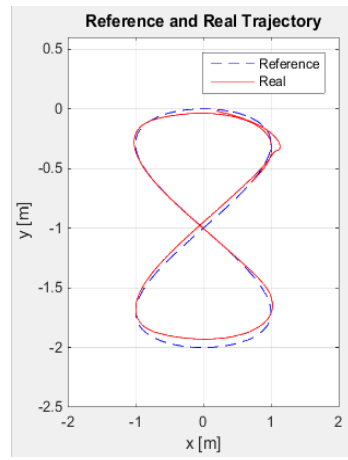

(a)

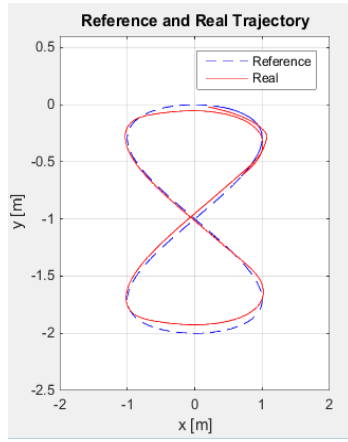

(c)

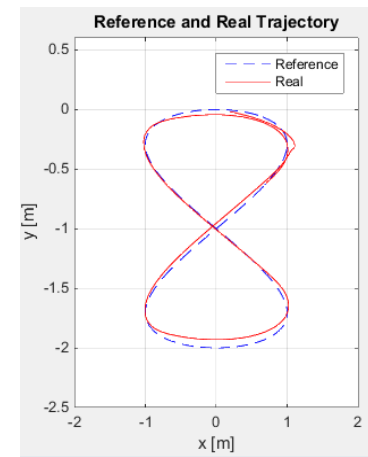

(b)

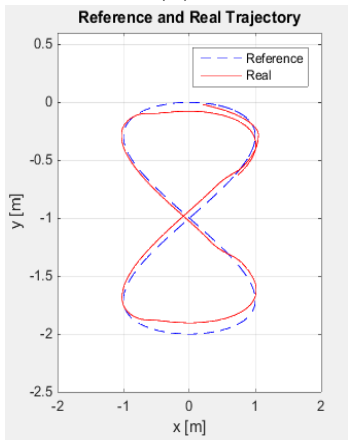

(d)
Figure 5. Reference eight-shaped trajectory and real trajectory of mobile robot using dynamic controller based on PID at different strength of dynamic uncertainty. a) No uncertainty b) $10 \%$ uncertainty c) $30 \%$ uncertainty d) $50 \%$ uncertainty 


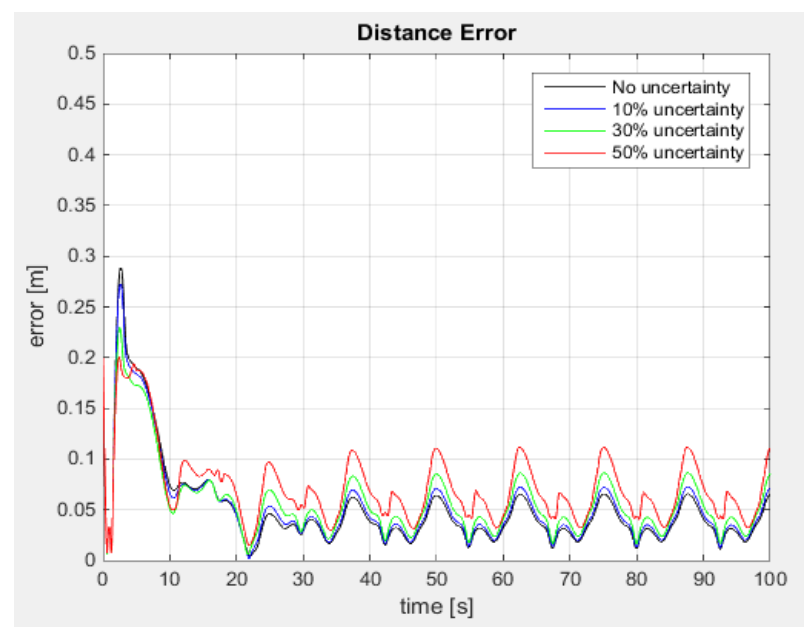

Figure 6: Distance error of trajectory tracking control using dynamic controller based on PID at different strength of dynamic uncertainty for eight-shaped trajectory

Table 2: IAE of trajectory tracking at different strength of dynamic uncertainty for both PID and SMC controllers

\begin{tabular}{|c|c|c|}
\hline \multirow{2}{*}{ Case } & \multicolumn{2}{|c|}{ IAE, $\mathrm{m}$} \\
\cline { 2 - 3 } No & PID & SMC \\
\hline $\begin{array}{c}\text { Uncertainty } \\
\mathbf{1 0 \%}\end{array}$ & 5.0530 & 2.1922 \\
\hline $\mathbf{3 0 \%}$ & 5.2772 & 2.2130 \\
\hline $\mathbf{5 0 \%}$ & 5.7447 & 2.3714 \\
\hline
\end{tabular}

\section{CONCLUSION}

A dynamic controller using SMC technique for trajectory tracking control of autonomous mobile robot is developed in MATLAB. The developed trajectory tracking controller is divided into two parts, kinematic controller and dynamic controller. The kinematic controller is used to generate a desired velocity command based on current robot position and desired position. Meanwhile, dynamic controller is used to compensate the effect of robot dynamics by minimizing the error between the velocity of mobile robot and the desired velocity generated by kinematic controller. The stability of both dynamic and kinematic controller is verified using Lyapunov stability theory. The performance of trajectory tracking control for proposed dynamic controller based on SMC is compared against dynamic controller based on PID under the presence of different strength of dynamic uncertainties. Simulation results show the proposed dynamic controller based on SMC give better performance in trajectory tracking control in comparison to dynamic controller based on PID.

\section{REFERENCES}

[1] A. P. Aguiar and J. P. Hespanha, "Trajectorytracking and path-following of underactuated autonomous vehicles with parametric modeling uncertainty," IEEE Transactions on Automatic Control, vol. 52, pp. 1362-1379, 2007.
[2] F. N. Martins, W. C. Celeste, R. Carelli, M. Sarcinelli-Filho, and T. F. Bastos-Filho, "An adaptive dynamic controller for autonomous mobile robot trajectory tracking," Control Engineering Practice, vol. 16, pp. 1354-1363, 2008.

[3] Z.-P. Jiang and H. Nijmeijer, "A recursive technique for tracking control of nonholonomic systems in chained form," Automatic Control, IEEE Transactions, vol. 44, pp. 265-279, 1999.

[4] S. Sun, "Designing approach on trajectory-tracking control of mobile robot," Robotics and ComputerIntegrated Manufacturing, vol. 21, pp. 81-85, 2005.

[5] H. Wang, B. Salatin, G. G. Grindle, D. Ding, and R. A. Cooper, "Real-time model based electrical powered wheelchair control," Medical engineering \& physics, vol. 31, pp. 1244-1254, 2009.

[6] Y. Kaubaa, M. Boukattaya, and T. Dammak, "Adaptive Sliding-Mode Dynamic Control For Path Tracking of Nonholonomic Wheeled Mobile Robot," Jounal of Automation and System Engineering, vol. 9, pp. 119-131, 2015.

[7] T. Das and I. N. Kar, "Design and implementation of an adaptive fuzzy logic-based controller for wheeled mobile robots," IEEE Transactions on Control Systems Technology, vol. 14, pp. 501-510, 2006.

[8] Q. Xu, J. Kan, S. Chen, and S. Yan, "Fuzzy PID based trajectory tracking control of mobile robots and its simulation in simulink," International Journal of Control and Automation, vol. 7, pp. 233244, 2014.

[9] D. Gu and H. Hu, "Wavelet neural network based predictive control for mobile robots," in Systems, Man, and Cybernetics, 2000 IEEE International Conference on, pp. 3544-3549, 2000.

[10] A. Onat and M. Ozkan, "Dynamic adaptive trajectory tracking control of nonholonomic mobile robots using multiple models approach," Advanced Robotics, vol. 29, pp. 913-928, 2015/07/18 2015.

[11] F. Garelli, L. Gracia, A. Sala, and P. Albertos, "Sliding mode speed auto-regulation technique for robotic tracking," Robotics and Autonomous Systems, vol. 59, pp. 519-529, 7// 2011.

[12] C. De La Cruz and R. Carelli, "Dynamic modeling and centralized formation control of mobile robots," in IEEE Industrial Electronics, IECON 2006-32nd Annual Conference on, pp. 3880-3885, 2006.

[13] Majid, N. A., Mohamed, Z. and Basri, M. A. M. Velocity Control of a Unicycle Type of Mobile Robot Using Optimal PID Controller. Jurnal Teknologi.78(7-4), 7-14, 2016. 